\title{
Revisión del estado actual de la cirugía de reasignación genital en pacientes con disforia de género enfocada en el contexto colombiano
}

\section{Review of the Current Status of Genital Reassignment Surgery in Patients with Gender Dysphoria within the Colombian Context}

José Miguel Silva ${ }^{1}$ Lynda Torres Castellanos ${ }^{1}$ Alejandra Bravo-Balado ${ }^{2}$ Angie Puerto Niño ${ }^{3}$ Juan David Urazán ${ }^{3}$ Andrés Camilo Cardozo Alarcón ${ }^{4}$

\footnotetext{
1 Unidad de Urología, Hospital Universitario San Ignacio y Facultad de Medicina, Pontificia Universidad Javeriana, Bogotá D.C., Colombia

2 Departamento de Urología, Hospital Universitario Fundación Santa Fe de Bogotá y Facultad de Medicina, Universidad de los Andes, Bogotá D.C., Colombia

3 Facultad de Medicina, Pontificia Universidad Javeriana, Bogotá D.C., Colombia

${ }^{4}$ Servicio de Psiquiatría y Salud Mental, Hospital Universitario San Ignacio y Facultad de Medicina, Pontificia Universidad Javeriana, Bogotá D.C., Colombia
}

Address for correspondence José Miguel Silva, MD, Urología y Medicina Sexual, Unidad de Urología, Hospital Universitario San Ignacio y Facultad de Medicina, Pontificia Universidad Javeriana, Carrera 7 No.40 - 62, Unidad de Urología, piso 8, 110231 (e-mail: jose.silva@javeriana.edu.co).

Urol Colomb 2019;28:196-203.

\section{Resumen \\ Palabras Clave \\ - disforia de género \\ - cirugía de reasignación genital \\ - transexualismo \\ - legislación \\ - calidad de vida \\ - Colombia}

La disforia de género o transexualismo es una condición que comprende a las personas que padecen profundo malestar o rechazo hacia el sexo biológico por cuanto se identifican con el sexo opuesto. Por lo tanto, se asocia a disfunción biopsicosocial y compromiso severo de la calidad de vida. En Colombia se han producido notables avances de índole constitucional y legislativa en las dos últimas décadas, los cuales son discutidos en la presente revisión. En el tratamiento multidisciplinario de los pacientes transexuales, el último paso corresponde a la cirugía de reasignación genital. El propósito de esta revisión es investigar el estado actual de la cirugía de reasignación genital enfocada en el contexto nacional.

Gender dysphoria or transsexualism is a condition that includes people who suffer from deep discomfort or rejection of biological sex because they identify with the opposite sex. Therefore, it is associated with biopsychosocial dysfunction and severe compromise of their quality of life. In Colombia there have been notable advances of a constitutional and legislative nature in the last two decades, which are discussed in the present review. In the multidisciplinary treatment of transgender patients, the last step corresponds to genital reassignment surgery. The purpose of this review is to investigate the current status of genital reassignment surgery within the national context. received

March 24, 2018

accepted

May 3, 2018
DOI https://doi.org/ 10.1055/s-0038-1657773. ISSN 0120-789X. eISSN 2027-0119.
Copyright (c) 2019, Sociedad Colombiana License terms de Urología. Publicado por Thieme Revinter Publicações Ltda., Rio de Janeiro, Brazil. Todos los derechos reservados.

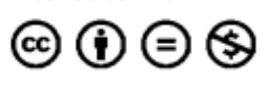




\section{Introducción}

La disforia de género es el malestar profundo originado por la discrepancia entre la identidad de género y el sexo asignado de acuerdo con los caracteres sexuales al nacer. Dicho de otra manera, se trata de la disconformidad entre el sexo psicológico con el cual una persona se define a sí misma y las características sexuales primarias y secundarias. ${ }^{1}$

Hasta la fecha no se dispone de estudios epidemiológicos formales sobre incidencia y prevalencia, por lo que, en general, los esfuerzos para llegar a estimaciones realistas encuentran enormes dificultades. ${ }^{1}$ Sin embargo, la incidencia descrita corresponde a 1 en 60.000 , lo que parece constante en diferentes razas y culturas. Los transexuales del sexo masculino al femenino (hombre mujer), son cuatro veces más frecuentes que los transexuales del sexo femenino al masculino (mujerhombre). ${ }^{2,3}$ Por tratarse de una condición de baja frecuencia, es poco lo que se conoce sobre el impacto del tratamiento, incluida la cirugía de reasignación genital (CRG), en la población colombiana. Un estudio publicado recientemente en Colombia estudió 4 pacientes transexuales sometidos a CRG hombre-mujer y se encontró que la calidad de vida en ese grupo de pacientes fue calificada como satisfactoria. Sin embargo, el aspecto de relaciones interpersonales fue calificado como pobremente favorable, explicado por las actitudes de intolerancia en la sociedad que causan un alto y permanente grado de sufrimiento en esas personas. ${ }^{4}$

El objetivo de esta revisión es dar a conocer el estado actual de la CRG en pacientes con disforia de género. Nuestro mayor interés es reunir la mejor evidencia actualmente disponible en el tema enfocados en el contexto colombiano, además de resaltar la importancia del tratamiento, puesto que él garantiza la salud de dichos pacientes al tiempo que respeta la dignidad y el derecho a desarrollar una adecuada identidad sexual, lo que, en consecuencia, reduce el sufrimiento que esa condición trae consigo dado su estigma social.

\section{Datos históricos y definición actual de disforia de género}

Aunque la primera operación genital documentada data de 1931 en el caso de Lili Elbe, es posible afirmar que inicialmente los conceptos terapéuticos persiguieron ajustar la identidad sexual al sexo biológico, por lo que los tratamientos efectuados antes de 1953 fueron de índole psiquiátrica.

En 1950, el sexólogo David Cauldwell utilizó por primera vez el término "transexual" en un texto que calificó la condición como una psicopatía.

Pocos años después, en 1953, se intervino una paciente en Dinamarca de vaginoplastia, hecho que fue muy difundido y causó gran revuelo. Para ese entonces, Harry Benjamin, endocrinólogo alemán radicado en Estados Unidos, se refirió a la diferencia entre transexualidad y travestismo. A él se le atribuye el haber popularizado el término, además de que calificó la causa como endocrina y no mental, lo que permitió defender la cirugía como la forma de ajustar el sexo biológico a la identidad sexual, dado que las intervenciones psicoterapéuticas que pretendían lo contrario no resultaban exitosas. En el año 1966, Benjamin formalizó los criterios diagnósticos para el transexualismo. ${ }^{2}$

En 1968, el psicoanalista Robert Stoller, publicó el libro "Sex and Gender" en el que se refiere a las variantes de la sexualidad humana, y entre ellas al transexualismo, como la convicción de una persona de sexo biológico normal de pertenecer al sexo opuesto y lo diferencia del travestismo y la homosexualidad.

Posteriormente, Fisk en 1974, se refiere al concepto de género en el que se interrelacionan un número amplio de factores biológicos, psicológicos y psicosociales. Enumera factores tales como: la carga cromosómica, al sexo asignado al nacimiento y el de crianza, la morfología genital externa e interna, los factores endocrinológicos prenatales y postnatales, además del comportamiento. Con los estudios de Fisk aparece el concepto de "disforia de género" cuya definición incluye sentimientos de insatisfacción, ansiedad, inquietud e incomodidad. ${ }^{5}$

El trastorno de identidad de género llegó por primera vez a la nosología psiquiátrica en 1980 en el DSM-III, y las versiones posteriores han subdividido y categorizado de diferentes maneras ese espectro de condiciones, hasta alcanzar la disforia de género del actual DSM-5. Para formalizar el diagnóstico, la actual clasificación exige que un paciente debe haber tenido durante seis meses o más una marcada incongruencia entre el género asignado al nacimiento y el género que siente o expresa como propio, lo que debe acompañarse además de algún deterioro funcional $o$ de angustia clínicamente significativa (-Cuadro 1). ${ }^{6,7}$

A pesar de que la etiopatogenia no ha sido totalmente dilucidada, se han planteado diversas teorías, algunas basadas en el papel de los factores ambientales y psicológicos externos, así como la nutrición. En las últimas dos décadas, el grupo Swaab en Amsterdam, describió una base neurológica para la identidad de género centrada en la diferenciación morfológica sexual del cerebro en la que se apoyan las teorías del dismorfismo sexual. Son ellas las áreas cerebrales localizadas en el sistema límbico, tales como los núcleos interticial del hipotálamo anterior, ventromedial y supraquiasmático, amígdala y estría terminales. Se han descrito diferencias relacionadas con el volumen de la subdivisión central del núcleo de la estría terminal (área cerebral esencial para el comportamiento sexual) el cual es mayor en hombres que en mujeres. Esos hallazgos son similares en transexuales, por cuanto, en los transexuales hombre-mujer, esa área suele tener el mismo tamaño de las personas del sexo femenino y viceversa. ${ }^{8,9}$ Otra zona relacionada con la identidad de género corresponde al núcleo uncinado del hipotálamo. ${ }^{10,11}$ Ninguna de esas variables neuroanatómicas tienen relación con la orinetación sexual. 
Cuadro 1 Posibles manifestaciones de la incongruencia entre el género asignado al nacimiento y el género que siente y expresa como propio

\begin{tabular}{|l|}
\hline $\begin{array}{l}\text { El diagnóstico de Disforia de Género o Transexualismo en adolescentes y adultos, requiere al menos dos de estos criterios, } \\
\text { de acuerdo con el Manual Diagnóstico y Estadístico de los Trastornos Mentales DSM-5 }\end{array}$ \\
\hline 1. Marcada incongruencia entre el género experimentado como propio y sus características sexuales primarias o secundarias. \\
\hline $\begin{array}{l}\text { 2. Fuerte deseo de deshacerse de sus características sexuales primarias o secundarias (o de impedir su desarrollo) a causa de } \\
\text { dicha incongruencia. }\end{array}$ \\
\hline 3. Fuerte deseo de tener las características sexuales primarias y secundarias del sexo opuesto. \\
\hline 4. Fuerte deseo de ser una persona de otro género. \\
\hline 5. Fuerte deseo de ser tratado como una persona con otro género. \\
\hline 6. Fuerte creencia de que tiene los sentimientos o reacciones de una persona con otro género. \\
\hline
\end{tabular}

\section{Legislación colombiana respecto a la disforia de género y la cirugía de reasignación genital}

En Colombia, se han dado avances importantes a través de la historia en la esfera constitucional y legislativa. Lo más destacado en materia legislativa se presenta en la - Tabla 1.

\section{Tratamiento de la disforia de género}

Las opciones terapéuticas actuales abarcan cinco etapas distintas, son estas: diagnóstico, psicoterapia, experiencia de la vida real en un rol de género congruente con la identidad, terapia hormonal y, finalmente, la CRG. ${ }^{16}$

Existe controversia con respecto al tratamiento con psicoterapia frente a la CRG. Muchos especialistas coinciden en que la cirugía se convierte en el tratamiento de elección para los casos de disforia de género, debido a que la psicoterapia como única intervención no se ha mostrado efectiva. $^{17}$

La frecuencia de la CRG depende de la aceptación cultural y varía entre las diferentes poblaciones, desde 1:11.900 a
1:45.000 para la reasignación hombre-mujer y de 1:30.400 a 1: 200.000 para la de mujer-hombre. ${ }^{16,18}$

\section{Cirugía de reasignación genital hombre-mujer}

El objetivo es crear un área perineo-vaginal con apariencia y funcionalidad femeninas sin complicaciones asociadas. La cirugía persigue obtener una uretra corta, un chorro urinario hacia abajo para que la persona pueda orinar sentada, ausencia de estenosis o fístulas, una neovagina elástica sin vello y con profundidad de al menos $10 \mathrm{~cm}$, diámetro de $30 \mathrm{~mm}$ y sensibilidad suficiente para el estímulo erógeno durante la actividad sexual. ${ }^{19}$

Actualmente el colgajo peno-escrotal invertido es más aceptado para la CRG hombre-mujer que los injertos de piel. Eso se debe a que tiende a contraerse menos, puede tener inervación local y no tiene vello. Sin embargo, en ocasiones, no hay piel suficiente para la neocavidad, dejando la comisura anterior más amplia y con exposición del clítoris; eso se puede subsanar integrando un injerto al extremo distal de la piel del pene. ${ }^{20}$

Tabla 1 Legislación colombiana

\begin{tabular}{|l|l|}
\hline Ley & Avance \\
\hline - Sentencia T-622/14 & $\begin{array}{l}\text { Establece la obligación de las Entidades Promotoras de Salud (EPS) de cubrir las } \\
\text { cirugías de reasignación genital basada en el derecho a la identidad sexual. Se } \\
\text { entiende como una vulneración el no cumplir con las acciones necesarias para tal } \\
\text { efecto ante un consentimiento informado, cualificado y persistente. Asimismo, las } \\
\text { obliga a brindar asesoría a los menores de edad y a sus padres acerca de las } \\
\text { consecuencias de los tratamientos y cirugías de reasignación, y a consultar al niño } \\
\text { acerca de la decisión final adoptada. }{ }^{12}\end{array}$ \\
\hline $\begin{array}{l}\text { - Artículo 10 de la Ley } 48 \text { de 1993. } \\
\text { - Sentencia C-006/16 }\end{array}$ & $\begin{array}{l}\text { Dispone que “las mujeres transgénero no son destinatarias de las normas sobre } \\
\text { servicio militar obligatorio y que, bajo el principio de autonomía y libre desarrollo de } \\
\text { la personalidad, el autoreconocimiento es suficiente para ser exoneradas del } \\
\text { servicio." }\end{array}$ \\
\hline - Sentencia T-062/11 & Se refiere al respeto y buen trato en entidades carcelarias. ${ }^{14}$ \\
\hline - Sentencia 594 de 1993 y 977 de 2012 & Permite cambiar el nombre de nacimiento. ${ }^{15}$ \\
\hline - Decreto 1227 de 4 de junio de 2015 & $\begin{array}{l}\text { Dicta el protocolo y las reglas para la modificación del registro civil, con el fin de } \\
\text { evitar la violación de múltiples derechos fundamentales, el trato desigual y eliminar } \\
\text { la tendencia hacia la patologización de la identidad de género. }{ }^{15}\end{array}$ \\
\hline
\end{tabular}


Adicionalmente, para asegurar la mejor longitud vaginal, es necesario anclar la porción superior de la neovagina al ligamento sacroespinoso, lo que además procura evitar el prolapso. ${ }^{20}$ Entre las complicaciones más comunes de esa técnica figuran el sangrado proveniente del cuerpo esponjoso, la estenosis uretral, la pérdida de la amplitud vaginal, la fístula rectovaginal y el crecimiento de vello en la vagina.

La dilataciones vaginales posoperatorias con conformadores de tallas crecientes deben mantenerse por tiempo indefinido para lo que se debe indicar el uso de lubricantes, de la misma manera que para la actividad sexual con penetración. ${ }^{20}$ No es raro que se requieran algunas intervenciones adicionales para obtener todos los objetivos de la cirugía.

La vaginoplastia con segmento intestinal tiene ventajas cuando de longitud, textura y apariencia similar a la vagina se trata, además de que brinda lubricación natural. El segmento intestinal que con más frecuencia se utiliza es el sigmoide, aunque también, es posible emplear colon derecho e íleon. Con esa técnica, las complicaciones se asocian a la cirugía intestinal en el posoperatorio inmediato y tienen relación con la producción excesiva de moco, colitis ulcerativa, mucocele y neoplasias. El uso de esa técnica ha aumentado en varios centros médicos. ${ }^{17,20}$

El método de Eld consiste en el uso del glande para la clitoroplastia e incluye el paquete neurovascular que se encuentra entre la fascia de Buck y el cuerpo cavernoso. Los desenlaces de esa técnica han demostrado alta sensibilidad y satisfacción sexual. ${ }^{17}$ La plastia de los labios mayores se obtiene a partir de la piel del escroto. Con relativa frecuencia se necesitan procedimientos menores adicionales de retoque para mejorar la apariencia. ${ }^{19,20}$ Los labios menores se obtienen del tejido disponible después de completada la vaginoplastia. La plastia de los labios menores se puede elaborar con piel del pene e incluye el segmento mucocutaneo del prepucio para rodear el neoclítoris procedente del glande remodelado ( - Fig. 1). Las estructuras anteriormente nombradas son el mayor reto de la CRG. ${ }^{17,19-21}$

\section{Cirugía de reasignación genital mujer-hombre}

El objetivo de la faloplastia es crear un pene cosméticamente satisfactorio con neouretra que se abra en la punta del pene y que permita la actividad sexual con penetración. ${ }^{19}$ Se obtienen colgajos de antebrazo, peroné, muslo y dorsal ancho. ${ }^{17,22} \mathrm{~A}$ pesar de que no hay una técnica que logre perfectamente los todos los objetivos de la cirugía, el colgajo más comúnmente utilizado es el de antebrazo radial. Esa cirugía implica técnica microquirúrgica, alta estancia hospitalaria y múltiples cirugías de revisión ( - Fig. 2).

La complicación más frecuente consiste en la fístula urinaria que se presenta hasta en un 30\% de los casos, o estrechez uretral en la anastomosis de la porción uretral del neofalo y la parte fija de la uretra femenina. ${ }^{23,24}$ Hasta la fecha, no existe gran evidencia sobre el colgajo de dorsal ancho. Con el colgajo abdominal infraumbilical se suele conseguir un neofalo de poca sensibilidad que ofrece dificultad para la micción y la penetración. ${ }^{19}$ También es parte del procedimiento realizar vaginectomía durante la cirugía mayor y la escrotoplastia con colgajos a partir de los labios mayores.

\section{Prótesis peneana}

La idea del neofalo es crear un pene cosméticamente aceptable que permita el rol masculino de orinar de pie y la rigidez suficiente para la penetración; allí radica el desafío. El método ideal de implantación es motivo de controversia; el anclaje, la estabilidad y la prevención de erosiones son metas difíciles de lograr. Un estudio publicado recientemente por Falcone y cols., ${ }^{25}$ incluyó 247 pacientes desde 2001 a octubre de 2015, quienes fueron implantados

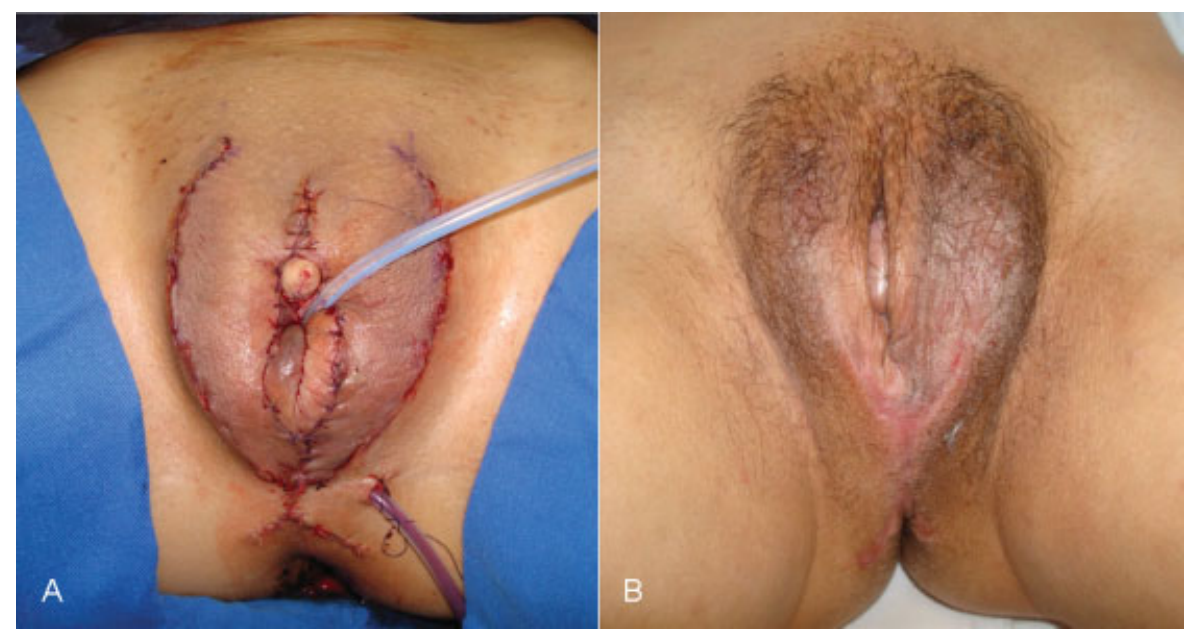

Fig. 1 Cirugía de reasignación hombre-mujer en (A) Postoperatorio inmediato y (B) Postoperatorio tardío. 


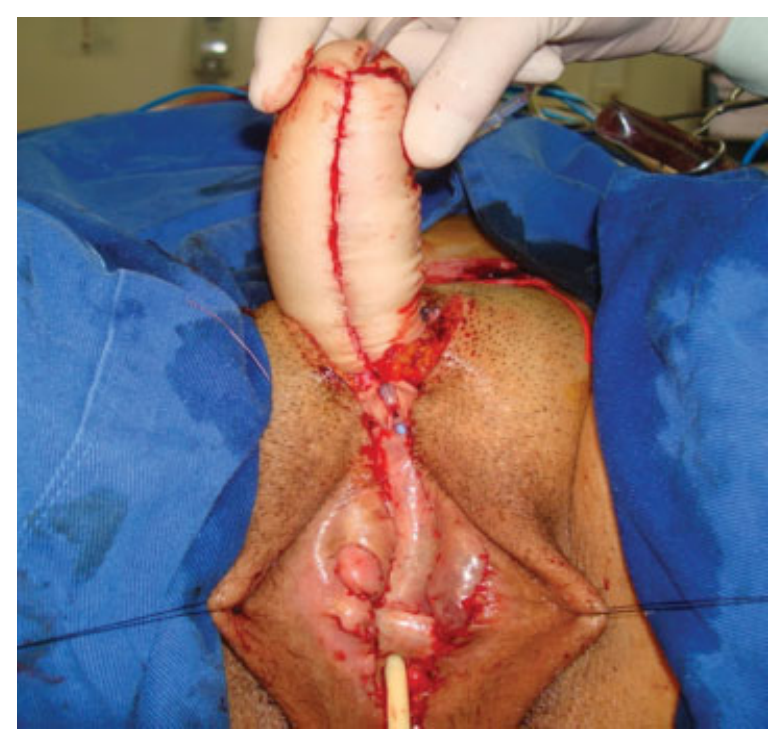

Fig. 2 Neofalo a partir de colgajo de antebrazo radial en cirugía de reasignación mujer-hombre.

con prótesis de pene inflables. De ellos, 157 tenían colgajo de arteria radial y 90 colgajo púbico infraumbilical. El procedimiento comprendió dos etapas: la primera, que consistió en la inserción del reservorio más la creación del glande e inserción de la prótesis testicular en el labio mayor ipsilateral a la mano dominante. La segunda etapa, realizada tres meses después, constó de una incisión inguinal, exposición del aspecto medial de la sínfisis del pubis, anclaje de la prótesis con aguja en J poliéster 0 en dos ramos paralelos cada cilindro separado por $2 \mathrm{~cm}$, dilatación del neofalo con dilatador de Hegar hasta $18 \mathrm{Fr}$ y la colocación de un injerto de Dacron para asegurar que estuvieran lejos de la piel. La longitud del falo se determinó desde la punta hasta el pubis. Los cilindros se instalaron con el dilatador de Furlow. Posteriormente, se retiró la prótesis testicular para dejar la bomba en ese lugar. Los cilindros quedaron semi-inflados por una semana. La prótesis testicular se instaló en el labio controlateral. A las seis semanas del procedimiento iniciaron la manipulación del dispositivo. La edad promedio de los pacientes fue de 38 años (rango 21-69). Al 63\% se les implantó un cilindro con longitud promedio de $16,9 \mathrm{~cm}$. El seguimiento fue de 20 meses. El 56\% (140 pacientes), mantuvieron la prótesis en su sitio original y los restantes necesitaron revisión.

El tiempo útil esperado con las prótesis fue de tres años, por cuanto la vida útil en los neofalos tiende a ser menor. Las causas de falla fueron: ruptura del cilindro en el 69\%, aneurisma del cilindro $19 \%$ y en el $12 \%$ defectos en las conexiones. Un total de 12 pacientes requirieron múltiples cirugías de revisión. A cinco años, la supervivencia del implante fue del $78 \%$. El factor más asociado a la necesidad de revisión fue la faloplastia con colgajo púbico inflaumbilical. No hubo factores especialmente asociados con infección o falla mecánica. Un total de 97 pacientes
(77\%) lograron penetración, 77 (61\%) lograron orgasmo, 97 (88\%) se sintieron completamente satisfechos con el resultado funcional y la satisfacción de la pareja fue del $60 \%$. Esos son resultados excepcionales y propios de un centro de alta experiencia y, por lo tanto, son difíciles de replicar.

\section{Resultados estéticos de la cirugía de reasignación genital}

Para la evaluación de los resultados posoperatorios se tienen en cuenta dos tipos de desenlaces: 1) estéticos y 2) funcionales (satisfacción sexual y función miccional). 26,27 En el tratamiento de los hombres con disforia de género, la construcción quirúrgica de una neovagina mediante una vaginoplastia es la etapa final en la transición al género deseado. Como ya se ha dicho, el objetivo quirúrgico consiste en crear una neovagina que sea lo más funcional posible, con una apariencia muy similar a una vagina normal. Sin embargo, aún no es claro qué tan satisfechas están las mujeres transgénero con los resultados funcionales $\mathrm{y}$ estéticos de la vaginoplastia. ${ }^{20}$

En 2015 Buncamper y cols., ${ }^{28}$ publicaron un estudio retrospectivo a partir de los datos obtenidos de 49 mujeres transgénero sometidas a vaginoplastia mediante la técnica de “inversión de la piel del pene." Los investigadores aplicaron a los pacientes el índice de función sexual femenina (Female Sexual Function Index - FSFI) y solicitaron responder un cuestionario de 16 preguntas Short Questionnaire for Self-Evaluation of Vaginoplasty (SQSV) -, para establecer el grado de satisfacción estética y funcional de las pacientes. Adicionalmente, fotografías de los genitales fueron valoradas por un panel independiente de evaluadores para objetivizar los resultados estéticos de la vaginoplastia. El panel de evaluadores fue conformado por dos cirujanos plásticos, dos ginecólogos y cuatro personas no relacionadas con el campo de la medicina. Los evaluadores calificaron la apariencia estética de los genitales y respondieron si encontraban suficientemente femenina la neovagina en cada caso. En general, el panel puntuó los resultados estéticos con 6/10. Los evaluadores no médicos otorgaron puntajes notablemente más bajos que el resto del panel. En el 68\% de los casos, calificaron la apariencia genital lo suficientemente femenina y sugirieron cirugías correctivas en el $58 \%$ de los sujetos. ${ }^{20}$

Los investigadores encontraron además que, aunque las pacientes calificaron en promedio con 8/10 los resultados estéticos posoperatorios, el $56 \%$ se encontraba sexualmente disfuncional de acuerdo con el (FSFI), en algunos casos, por no ser sexualmente activas o debido a problemas de lubricación e incomodidad. ${ }^{20}$

Por otro lado, Hess y cols ${ }^{29}$ entrevistaron a 119 pacientes sometidos a CRG para evaluar la satisfacción subjetiva postoperatoria mediante un cuestionario de 11 preguntas. Los investigadores encontraron una alta tasa de satisfacción con el resultado estético y más del 70\% informó estar conforme con el resultado final. 
Los pacientes y los cirujanos han dado cada vez más importancia al resultado estético de la CRG. A pesar, de que existen estudios que sugieren una alta satisfacción con el resultado estético final de la CRG por parte de las pacientes, pocos trabajos han intentado ser objetivos con los resultados. ${ }^{30,31}$

\section{Calidad de vida, satisfacción sexual y funcionalidad de la micción}

Como se comentó previamente, la CRG en las mujeres transexuales incluye vaginoplastia y neoclitoroplastia. La estimulación del clítoris es uno de los factores cruciales para lograr el orgasmo. La sensibilidad erógena y táctil del neoclítoris en la CRG fue descrita por primera vez en 1978 por Wesser. ${ }^{23}$ La mayoría de las técnicas obtienen el colgajo para el neoclítoris a partir de la sección dorsal proximal del glande. ${ }^{32,33}$

En 2016 Sigurjónsson y cols, ${ }^{34}$ midieron la sensibilidades táctil y vibratoria mediante monofilamentos SemmesWeinstein y un biotesiómetro, respectivamente. Se incluyeron 22 pacientes con seguimiento promedio de 37 meses posoperatorios. Adicionalmente, se proporcionó un cuestionario para evaluar la percepción de su imagen corporal, orgasmo, dolor y la satisfacción general con la cirugía. Los investigadores establecieron que el umbral táctil promedio para el clítoris fue de $12,5 \mathrm{~g} / \mathrm{mm}^{2}$ y el umbral vibratorio promedio de $0,3 \mathrm{~mm}$. La mayoría de los participantes (86\%) experimentaron el orgasmo, no presentaron dolor y se mostraron satisfechos con la CRG. Los autores concluyeron que la formación del neoclítoris a partir del glande es una alternativa quirúrgica que conserva la sensibilidad táctil y la sensibilidad vibratoria necesarias para facilitar el orgasmo en muchas de las pacientes transgénero. Por lo demás, se requieren otros estudios que evalúen la satisfacción sexual de las pacientes transgénero tratadas con abordajes quirúrgicos diferentes al neoclítoris obtenido a partir del glande.

Por otra parte, existe un interés creciente respecto a los efectos de la CRG en el tracto urinario inferior. A pesar de eso, la mayoría de los estudios evalúan complicaciones urológicas a corto plazo y no efectúan seguimientos a largo plazo de la función miccional. En la CRG, la próstata no es eliminada, pero el tracto urinario inferior es modificado drásticamente ya que la uretra se reduce de longitud. ${ }^{35,36}$ En 2006 Kuhn y cols., ${ }^{37}$ evaluaron la presencia de alteraciones miccionales en 18 transexuales hombre-mujer, mediante las siguientes herramientas: 1) King's Health Questionnaire, 2) Escala visual análoga para el bienestar del paciente, 3) Ultrasonido perineal y transabdominal, 4) Tira reactiva de orina y uroflujometría. El estudio estableció, que los transexuales tienen un mayor riesgo de desarrollar trastornos miccionales - principalmente incontinencia urinaria de esfuerzo y vejiga hiperactiva - en comparación con los grupos de control de la misma edad. Se postula además que una de las principales razones para desarrollar patologías urinarias es el daño del nervio pudendo. ${ }^{29}$ Es de vital importancia discutir detalladamente durante la preparación acerca del alto riesgo de desarrollar molestias urinarias posoperatorias que conllevan las técnicas de reasignación genital.

\section{Psicopatología en pacientes sometidos a cirugía de reasignación genital}

La sola existencia de la disforia de género como entidad nosológica, está ampliamente debatida en la literatura psiquiátrica actual. Como se mencionó previamente, en el DSM-5 esos pacientes se caracterizan por sentir incongruencia entre el sexo que les fue asignado al nacer y el género que perciben como propio. ${ }^{6}$ Sin embargo, muchos autores argumentan que esas personas están dentro del espectro de la normalidad y la existencia del diagnóstico es en sí mismo discriminatorio. ${ }^{38}$ Esa corriente de pensamiento tiene tantos argumentos a su favor que incluso existen estudios que apoyan trasladar esa categoría por fuera del capítulo de trastornos mentales de la nueva versión de la Clasificación Internacional de Enfermedades. ${ }^{39,40}$ Sin embargo, los mismos autores admiten que existe una gran carga de enfermedad mental en esa población que debe estudiarse y atenderse oportunamente. ${ }^{38}$

En el año 2012, un equipo de instituciones en el Reino Unido hizo una encuesta de salud dirigida a aproximadamente 1.000 personas transgénero. ${ }^{41}$ Ese estudio mostró que el $10 \%$ de los participantes había estado hospitalizado alguna vez en una unidad de salud mental, el 38\% había sido diagnosticado con algún grado de ansiedad, el 55\% había sido diagnosticado con depresión, ${ }^{41}$ el $62 \%$ tenía riesgo de dependencia del alcohol, y el $84 \%$ había tenido alguna vez ideas suicidas. Un estudio similar en los Estados Unidos encuestó a más de 6.000 personas transgénero en el año $2008,{ }^{42}$ y los resultados tampoco fueron alentadores: el $8 \%$ de los participantes reportó consumo de alcohol u otras sustancias como una manera de afrontar el trato injusto percibido de la sociedad y el $41 \%$ manifestó haber tenido intentos suicidas.

Varios estudios publicados han intentado determinar el efecto de la CRG en el estado psicológico y psicosocial de los pacientes. ${ }^{43}$ Uno de los primeros estudios con ese objetivo, fue el de Mate-Kole y cols., ${ }^{44}$ realizado en el año 1990 en una población de 20 hombres transexuales que fueron llevados a CRG de forma temprana comparados con 20 que aún esperaban la cirugía, ambos con seguimiento a 2 años. Esos investigadores encontraron una mejoría estadísticamente significativa en las escalas de ansiedad, personalidad obsesiva-compulsiva, fobias, depresión y trastornos de somatización en los que recibieron tratamiento quirúrgico temprano. También reportaron mejoría en la actividad social y sexual, como también, mejores tasas de empleo. Asimismo, otros grupos de investigadores en diferentes momentos han encontrado un efecto positivo de la CRG sobre medidas de bienestar social, satisfacción sexual y múltiples indicadores de psicopatología, ${ }^{34,45}$ incluídos estudios de seguimiento desde la adolescencia hasta un año después de la CRG. ${ }^{46}$

Esa evidencia indica que debe existir un trabajo conjunto entre los profesionales de las diferentes especialidades para 
la atención integral de esos pacientes. Múltiples instituciones se han pronunciado en torno a ese tema, en especial la Asociación Profesional Mundial para la Salud del Transgénero (WPATH, por sus siglas en inglés), la cual propone en sus estándares de atención, que los profesionales de salud mental y los demás especialistas cumplan con determinados requisitos de competencia y tengan la posibilidad de desempeñar diferentes roles de evaluación con respecto a la salud mental, tratamientos hormonales, quirúrgicos y psicoterapia. ${ }^{47}$

\section{Conclusiones}

La CRG es uno de los pasos trascendentales en el tratamiento de la disforia de género. Aunque existen diversas técnicas quirúrgicas satisfactorias, no hay una perfecta que logre todos los objetivos propuestos desde una visión ideal. De todas maneras, al tener en cuenta la evidencia mostrada, se refuerza la idea de que el sistema de salud colombiano, fundamentado en la legislación, debe y puede garantizar el tratamiento quirúrgico oportuno de los pacientes transexuales, con el fin de asegurarles los mejores desenlaces globales que conduzcan a altos grados de satisfacción e incremento de la calidad de vida, en procura de respetar la dignidad humana de esas personas.

\section{Financiación}

Este artículo de revisión no contó con ningún tipo de financiación.

\section{Conflictos de interés}

Los autores declaran que no tienen ningún conflicto de interés en relación al presente artículo de revisión.

Agradecimientos

Este artículo de revisión no contó con ayuda externa.

\section{Referencias}

1 World Professional Association for Transgender Health. Standards of Care for the Health of Transsexual, Transgender, and Gender Nonconforming People - 7th Version. www.wpath.org. 2001:1132

2 Meyer W III, et al. The Harry Benjamin International Gender Dysphoria Association's standards of care for gender identity disorders, sixth version. World Prof Assoc Transgender Heal [online]. 2001

3 Bakker A, van Kesteren PJ, Gooren LJ, Bezemer PD. The prevalence of transsexualism in The Netherlands. Acta Psychiatr Scand 1993; 87(04):237-238

4 Silva Herrera JM, Chavarriaga Soto J, Feijoo Monroy S, et al. Calidad de vida, función del tracto urinario y salud sexual en cirugía de reasignación de sexo hombre a mujer. Urol Colomb 2016;25(02): 81-87. Doi: $10.1016 /$ j.uroco.2015.12.003

5 Tosh J. Psychology and Gender Dysphoria: Feminist and Transgender Perspectives. 1st ed. New York2016

6 American Psychiatric Association. Gender Dysphoria. Dsm 2013; $5: 2$

7 Atkinson SR, Russell D. Gender dysphoria. Aust Fam Physician 2015;44(11):792-796
8 Zhou JN, Hofman MA, Gooren LJ, Swaab DF. A sex difference in the human brain and its relation to transsexuality. Nature 1995;378 (6552):68-70

9 Kruijver FP, Zhou JN, Pool CW, Hofman MA, Gooren LJ, Swaab DF. Male-to-female transsexuals have female neuron numbers in a limbic nucleus. J Clin Endocrinol Metab 2000;85(05):2034-2041

10 Swaab DF, Chung WC, Kruijver FP, Hofman MA, Ishunina TA. Sexual differentiation of the human hypothalamus. Adv Exp Med Biol 2002;511:75-100, discussion 100-105

11 Garcia-Falgueras A, Swaab DF. A sex difference in the hypothalamic uncinate nucleus: relationship to gender identity. Brain 2008;131(Pt 12):3132-3146

12 Corte Constitucional. Sentencia T-622/14. http://www. corteconstitucional.gov.co/relatoria/2014/t-622-14.htm; 2014

13 Corte Constitucional. Norma Que Regula El Servicio Militar Obligatorio. http://www.corteconstitucional.gov.co/relatoria/ SENTENCIAS\%20DE\%20CONSTITUCIONALIDAD\%20DE\% 20INTERES16.php; 2016

14 Corte Constitucional. Tratamiento de Población Reclusa Que Pertenece a Minorias de Identidad Sexual. http://www. corteconstitucional.gov.co/relatoria/2011/t-062-11.htm; 2011

15 Ministerio de Justicia y del Derecho. Decreto 1227. https://www. minjusticia.gov.co/Portals/0/Ministerio/decreto\%20unico/\%23\% 20decretos/1.\%20DECRETO\%202015-1227\%20sexo\%20c\%C3\% A9dula.pdf; 2015

16 Amend B, Seibold J, Toomey P, Stenzl A, Sievert KD. Surgical reconstruction for male-to-female sex reassignment. Eur Urol 2013;64(01):141-149. Doi: 10.1016/j.eururo.2012.12.030

17 Selvaggi G, Bellringer J. Gender reassignment surgery: an overview. Nat Rev Urol 2011;8(05):274-282. Doi: 10.1038/nrurol.2011.46

18 De Cuypere G, Van Hemelrijck M, Michel A, et al. Prevalence and demography of transsexualism in Belgium. Eur Psychiatry 2007; 22(03):137-141. Doi: 10.1016/j.eurpsy.2006.10.002

19 Wroblewski P, Gustafsson J, Selvaggi G. Sex reassignment surgery for transsexuals. Curr Opin Endocrinol Diabetes Obes 2013;20 (06):570-574

20 Selvaggi G, Ceulemans P, De Cuypere G, et al. Gender identity disorder: general overview and surgical treatment for vaginoplasty in male-to-female transsexuals. Plast Reconstr Surg 2005;116(06):135e-145e

21 Trombetta C, Liguori G, Benvenuto S, et al. [Neourethroclitoroplasty according to Petrovic]. Urologia 2011;78 (04):267-273

22 Selvaggi G, Elander A. Penile reconstruction/formation. Curr Opin Urol 2008;18(06):589-597

23 Monstrey S, Hoebeke P, Selvaggi G, et al. Penile reconstruction: is the radial forearm flap really the standard technique? Plast Reconstr Surg 2009;124(02):510-518

24 Lumen N, Monstrey S, Goessaert AS, Oosterlinck W, Hoebeke P. Urethroplasty for strictures after phallic reconstruction: a singleinstitution experience. Eur Urol 2011;60(01):150-158

25 Falcone M, Garaffa G, Gillo A, Dente D, Christopher AN, Ralph DJ. Outcomes of inflatable penile prosthesis insertion in 247 patients completing female to male gender reassignment surgery. BJU Int 2018;121(01):139-144

26 Plemons ED. It is as it does: genital form and function in sex reassignment surgery. J Med Humanit 2014;35(01):37-55

27 Sigurjonsson H, Rinder J, Möllermark C, Farnebo F, Lundgren T. Male to female gender reassignment surgery: Surgical outcomes of consecutive patients during 14 years. JPRAS Open 2015;6:69-73

28 Buncamper ME, Honselaar JS, Bouman MB, Özer M, Kreukels BP, Mullender MG. Aesthetic and Functional Outcomes of Neovaginoplasty Using Penile Skin in Male-to-Female Transsexuals. J Sex Med 2015;12(07):1626-1634

29 Hess J, Neto R, Panic L, Rübben H, Senf W. Satisfaction with maleto-female gender reassignment surgery. Dtsch Arztebl Int 2014; 111(47):795-801 
30 Eldh J. Construction of a neovagina with preservation of the glans penis as a clitoris in male transsexuals. Plast Reconstr Surg 1993; 91(05):895-900, discussion 901-903

31 Wesser DR. A single stage operative technique for castration, vaginal construction and perineoplasty in transsexuals. Arch Sex Behav 1978;7(04):309-323. Doi: 10.1007/BF01542040

32 von Szalay L. [Construction of a neo-clitoris in male-to-female transsexuals]. Handchir Mikrochir Plast Chir 1990;22(05):277-278

33 Fang RH, Chen CF, Ma S. A new method for clitoroplasty in maleto-female sex reassignment surgery. Plast Reconstr Surg 1992;89 (04):679-682, discussion 683

34 Sigurjónsson H, Möllermark C, Rinder J, Farnebo F, Lundgren TK. Long-Term Sensitivity and Patient-Reported Functionality of the Neoclitoris After Gender Reassignment Surgery. J Sex Med 2017; 14(02):269-273

35 Khouri RK, Young VL, Casoli VM. Long-term results of total penile reconstruction with a prefabricated lateral arm free flap. J Urol 1998;160(02):383-388

36 Bettocchi C, Ralph DJ, Pryor JP. Pedicled pubic phalloplasty in females with gender dysphoria. BJU Int 2005;95(01):120-124

37 Kuhn A, Hiltebrand R, Birkhäuser M. Do transsexuals have micturition disorders? Eur J Obstet Gynecol Reprod Biol 2007; 131(02):226-230

38 Morgan J. Trans* health: "diversity, not pathology". Lancet Psychiatry 2015;2(02):124-125. Doi: 10.1016/S2215-0366(15) 00022-X

39 Drescher J, Cohen-Kettenis PT, Reed GM. Gender incongruence of childhood in the ICD-11: controversies, proposal, and rationale. Lancet Psychiatry 2016;3(03):297-304. Doi: 10.1016/S22150366(15)00586-6
40 Robles R, Fresán A, Vega-Ramírez H, et al. Removing transgender identity from the classification of mental disorders: a Mexican field study for ICD-11. Lancet Psychiatry 2016;3(09):850-859. Doi: 10.1016/S2215-0366(16)30165-1

41 Mcneil J, Bailey L, Ellis S, Morton J, Regan M. Trans Mental Health Study 2012. 2012. https://www.gires.org.uk/wp-content/uploads/ 2014/08/trans_mh_study.pdf. Accessed November 25, 2017

42 Grant JM, Mottet LA, Justin Tanis J. with Jack Harrison Jody Herman DmL, Keisling M. Injustice at Every Turn. http://www. thetaskforce.org/static_html/downloads/reports/reports/ ntds_full.pdf. Accessed November 25, 2017

43 Heylens G, Verroken C, De Cock S, T'Sjoen G, De Cuypere G. Effects of different steps in gender reassignment therapy on psychopathology: a prospective study of persons with a gender identity disorder. J Sex Med 2014;11(01):119-126

44 Mate-Kole C, Freschi M, Robin A. A controlled study of psychological and social change after surgical gender reassignment in selected male transsexuals. Br J Psychiatry 1990;157:261-264http://www. ncbi.nlm.nih.gov/pubmed/2224377 AccessedOctober212017

45 Smith YL, Van Goozen SH, Kuiper AJ, Cohen-Kettenis PT. Sex reassignment: outcomes and predictors of treatment for adolescent and adult transsexuals. Psychol Med 2005;35(01):89-99

46 de Vries AL, McGuire JK, Steensma TD, Wagenaar EC, Doreleijers TA, Cohen-Kettenis PT. Young adult psychological outcome after puberty suppression and gender reassignment. Pediatrics 2014; 134(04):696-704

47 Coleman E, Bockting W, Botzer M, et al. Standards of Care for the Health of Transsexual, Transgender, and Gender-Nonconforming People, Version 7. Int J Transgenderism 2012;13(04):165-232. Doi: $10.1080 / 15532739.2011 .700873$ 